schen Lunge gegen den Lufteintritt relativ gross ist, so genügen die elastischen Kräfte, mit welchen der Thorax in seine natürliche Exspirationsstellung ïberzugehen strebt, nachdem der intrauterine Druck durch die Geburt beseitigt ist, nicht zur Entfaltung von Lunge und Thorax, sondern es müssen grössere Kräfte einwirken, wie sie durch die Inspirationsmuskeln oder durch künstliche Aufblasung entwickelt werden. Ist ferner die Lunge einmal lufthaltig geworden, so genügen wiederum die elastischen Kräfte derselben nicht, den Luftgehalt wieder völlig bis zur Atelectase auszutreiben. Jedenfalls zwingt uns nichts zu der Annahme, dass die erste Entfaltung der Lunge, sei es durch Inspiration oder durch Lufteinblasung, an der Thoraxwand selbst irgend eine bleibende Veränderung hervorrufe.

(Aus dem physiologischen Laboratorium in Zürich.)

\title{
Ueber Brechung bei schiefer Incidenz, mit besonderer Berücksichtigung des Auges, II. Theil.
}

(Vergl. dies Archiv Bd. XVIII. S. $443-455$ und Taf. VI.)

Von

\section{Hermann.}

\section{Die Periscopie dünner Linsen.}

Die Güte eines astigmatischen Bildes ist um so grösser, je kleiner der gegenseitige Abstand beider Brennlinien, d. h. die Entfernung $F_{2}-F_{1}$. Indessen muss man, um vergleichbare Werthe zu erhalten, auch die Gröss e des Bildes berücksicktigen, ähnlich wie bei gewöhnlichen Zerstreuungsbildern. Gleich grosse Zerstreuungskreise wirken um so störender je kleiner das Bild ist.

In meiner ersten Arbeit iiber den vorliegenden Gegenstand (1874) habe ich deshalb den Werth

$$
\frac{F_{2}-F_{1}}{F}
$$

worin F die centrale Brennweite, als ein reciprokes Maass der Güte des Bildes bei gegebenem Incidenzwinkel betrachtet. Es ergab sich für die dünne Linse 
Ueber Brechung b. schiefer Incidenz, m. besond. Berücksichtigung d. Auges. 371

$$
\frac{\mathrm{F}_{2}-\mathrm{F}_{1}}{\mathrm{~F}}=\frac{(\mathrm{n}-1) \sin ^{2} \varphi}{\mathrm{A}},
$$

worin $\mathrm{A}=\sqrt{\mathrm{n}^{2}-\sin ^{2} \varphi}-\cos \varphi ; d$. h. bei gegebener Brennweite $\mathrm{F}$ und gegebenem Incidenzwinkel $\varphi$ ist die Brennstrecke um so kürrer, je kleiner der Brechungsindex der Linse. Ferner ergab sich für eine geschichtete dünne Linse, von der Art der Krystalllinse, d. h. mit nach innen zunehmenden Indices und zunehmender Krümmung der Trennungsflächen, für gegebene äussere Begrenzung, gegebene Brennweite und gegebenen Incidenzwinkel eine kürzere Brennstrecke, als wenn die Linse bei sonst gleichen Verhältnissen homogen ist ${ }^{1}$ ).

Indessen ergiebt eine weitere Ueberlegung, dass die Grösse $\frac{F_{2}-F_{1}}{F}$ kein ausreichendes Maass ist für die Vergleichung der Periscopie. Man betrachte die der vorigen Arbeit beigegebene Figur 2 (Band XVIII. Tafel 6 dieses Archivs). Vermindert man bei gleichbleibender centraler Brennweite den Index der dünnen Linse LL, so werden allerdings die anf den Vectoren sichtbaren Abstände $\mathrm{F}_{2} \mathrm{~F}_{1}$ kleiner, wie ans obiger Betrachtung hervorgeht; jedoch rücken gleichzeitig die Puncte $F_{2}$ und $F_{1}$ der Linse näher, durch Aenderung der beiden gezeichneten Curven, welche den geometrischen Ort der beiden Brennlinien darstellen. Die Bilder selbst also werden gleichzeitig kleiner. Beruicksichtigt man dies, so ergiebt sich, dass man ein wirklich zuverlässiges Maass der Bildergiite erst erhält, wenn man den Werth

$$
\frac{\mathrm{F}_{2}-\mathrm{F}_{1}}{\mathrm{~F}_{2}}
$$

aufstellt, dessen Nenner der Grösse des Bildes proportional ist. Man findet aber jetzt für die duinne Linse

$$
\frac{\mathrm{F}_{2}-\mathrm{F}_{1}}{\mathrm{~F}_{2}}=\sin ^{2} \varphi
$$

d. h. die Giute des Bildes bei einer dünnen Linse ist lediglich vom Incidenzwinkel, und nicht von den optisehen Constanten der Linse abhängig. Auch für die geschichtete Linse findet man den Werth $\frac{F_{2}-F_{1}}{F_{2}}$ unabhängig von den optischen Constanten. Der Vortheil, welchen die bezeichnete Schichtung durch Verkürzung der Brenn-

1) Vgl. meine im ersten Theil, S. 443, citirten Arbeiten. 
strecke bei gegebenem Incidenzwinkel bietet, wird also genau dadurch aufgewogen, dass die Bilder durch Annäherung der Brennlinien entsprechend verkleinert werden. Dies gilt natürlich nur für die unendlich dủnne Linse.

\section{Die Periscopie dicker Linsen.}

In meiner ersten Arbeit konnte ich für dicke Linsen keine allgemeinen Ausdrücke aufstellen: Die im ersten Theil der vorliegenden Arbeit gegebenen Gleichungen liefern uns aber nunmehr hierzu die Grundlagen. Wir beschränken uns zunächst auf solche Strahlenbiundel, welche durch den optischen Mittelpunct der Linse hindurchgehen.

Der Zähler desjenigen Ausdrucks, welcher ein reciprokes Maass der Güte des Bildes liefert, ist wiederum $F_{2}-F_{1}$, und ergiebt sich aus den Gleichnngen 35 und 36. Der Nenner ist aber hier nicht gleich $\mathrm{F}_{2}$ zu nehmen, denn $\mathrm{F}_{2}$ zählt von der hinteren Linsenfläche ab, ist also kein Maass für die Grösse des Bildes. Letztere wird vielmehr gemessen durch den Abstand des zweiten Brennpunets (in welchem wir uns die auffangende Fläche denken) von der Axe, auf dem Leitstrahl gemessen. Wir haben demnach dem gebrochenen Leitstrahl MB in Figur 6 (Reproduction der Figur 6.

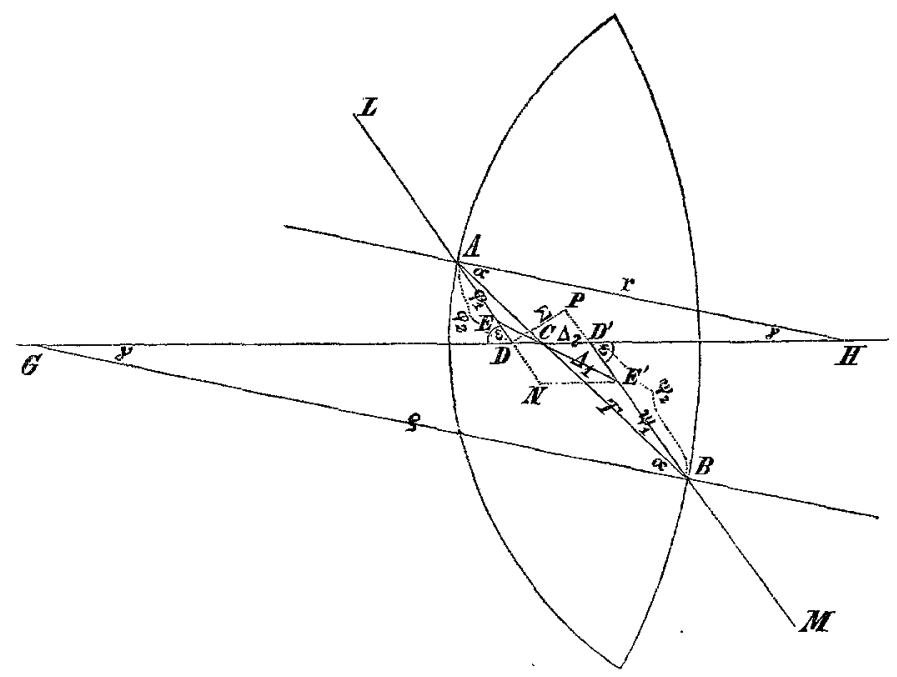


Ueber Brechung b. schiefer Incidenz, m. besond. Berücksichtigung d. Auges. 373

Figur 4 des 1. Theils) noch das Stück $\mathrm{BD}^{\prime}$ hinzuzufügen, und wenn wir dasselbe mit $S$ bezeichnen, so ist der Nenner des geforderten Ausdrucks $\mathrm{F}_{2}+\mathrm{S}$, also

$$
\frac{\mathrm{F}_{2}-\mathrm{F}_{1}}{\mathrm{~F}_{2}+\mathrm{S}}=\frac{1}{\mathrm{~B}}
$$

das reciproke Maass der absoluten Bildgüte $B$.

Der Werth von S ergiebt sich aus der Betrachtung des Dreiecks GBD' zu

$$
\left.\mathrm{S}=\varrho \cdot \frac{\sin \gamma}{\sin \varepsilon}=\frac{\varrho . \mathrm{T}}{\mathrm{n}(\mathrm{r}+\varrho)-\mathrm{AT}} \text { (nach Gleich. } 37 \mathrm{u} .39\right)
$$

also

$$
\mathrm{F}_{2}+\mathrm{S}=\frac{\varrho}{\mathrm{A}} \cdot \frac{\mathrm{n} \mathrm{r}}{\mathrm{n}(\mathrm{r}+\varrho)-\mathrm{AT}}
$$

Hiernach ist

oder

$$
\frac{1}{\mathrm{~B}}=\frac{\frac{\varrho}{\mathrm{A}} \cdot \frac{\mathrm{n} \mathrm{r}-\mathrm{AT}}{\mathrm{n}(\mathrm{r}+\varrho)-\mathrm{AT}}-\frac{\mathrm{N} \varrho}{\mathrm{A}} \cdot \frac{\mathrm{nr} \cos ^{2} \alpha-A T}{\mathrm{n}(\mathrm{r}+\varrho) \cos ^{2} \alpha-\mathrm{AT}}}{\frac{\varrho}{\mathrm{A}} \cdot \frac{\mathrm{n} \mathrm{r}}{\mathrm{n}(\mathrm{r}+\varrho)-\mathrm{AT}}}
$$

$$
\frac{1}{\mathrm{~B}}=\frac{1}{\mathrm{n} \mathrm{r}}\left\{\mathrm{nr}-\mathrm{AT}-\mathrm{N} \cdot \frac{\left(\mathrm{nr} \cos ^{2} \alpha-\mathrm{AT}\right)(\mathrm{n}(\mathrm{r}+\varrho)-\mathrm{AT})}{\mathrm{n}(\mathrm{r}+\varrho) \cos ^{2} \alpha-\mathrm{AT}}\right\} .
$$

In diesen Gleichungen ist (vgl. Seite 452 und 454 der vorigen Arbeit)

$$
\begin{aligned}
& \mathrm{A}=\mathrm{n} \cos \alpha-\sqrt{1-\mathrm{n}^{2} \sin ^{2} \alpha} \\
& \mathrm{N}=1-\mathrm{n}^{2} \sin ^{2} \alpha \\
& \mathrm{T}=(\mathrm{r}+\varrho) \cos \alpha-\sqrt{\mathrm{D}^{2}-(\mathrm{r}+\varrho)^{2} \sin ^{2} \alpha} \\
& \mathrm{D}=\mathrm{r}+\varrho-\mathrm{d} .
\end{aligned}
$$

Die Gleichung 45, welche in voller Strenge das reciproke Maass der absoluten Güte des Bildes für eine homogene Linse liefert, ist zu vergleichenden Untersuchungen über die Periscopie noch nicht ausreichend. Denn sie enthält nur den Winkel $\alpha$, d. h. den inneren Brechungswinkel des Strahlenbündels. Aendert man aber bei gegebener Incidenzschiefe des Bündels (welches stets durch den optischen Mittelpunct gehen soll) irgend etwas an der Linse, so ändert sich zugleich der Winkel $\alpha$. Statt des Winkels $\alpha$ muss also in die Gleichung 45 der Incidenzwinkel $\varepsilon, \mathrm{d}$. h. der Winkel zwischen einfallendem Bündel und Axe, eingeführt werden, der von den Constanten der Linse unabhängig ist. Die verwickelte Beziehung zwischen $\alpha$ und $\varepsilon$ wird durch die Gleichung 
40 oder 41 dargestellt. Man hätte also aus 40 (oder 41) und 45 $\alpha$ zu eliminiren, um die gesuchte Beziehang zwischen Güte des Bildes und Incidenzwinkel zu erhalten. Diese Beziehung fällt aber ungemein verwickelt aus.

Um eine iibersehbare Beziehung zu gewinnen, beschränken wir uns auf solche Winkel, deren Sinuspotenzen von der dritten ab vernachlässigt werden dürfen.

Mit dieser Beschränkung erbält man folgende Näherungswerthe:

$$
\begin{aligned}
\mathrm{A} & =(\mathrm{n}-1)\left(1+\frac{\mathrm{n}}{2} \sin ^{2} \alpha\right) \\
\mathbf{T} & =\mathrm{d}\left(1+\frac{\mathrm{r}+\varrho}{2 \mathrm{D}} \sin ^{2} \alpha\right) \\
\Delta \mathrm{T} & =(\mathrm{n}-1) \mathrm{d}\left[1+\frac{1}{2}\left(\mathrm{n}+\frac{\mathrm{r}+\varrho}{\mathrm{D}}\right) \sin ^{2} \alpha\right],
\end{aligned}
$$

und setzen wir zur Abkürzung für einen häufig vorkommenden Ausdruck

$$
\mathrm{Q}=\mathbf{n}(\mathbf{r}+\varrho)-(\mathbf{n}-1) \mathrm{d},
$$

so nimmt mit Einsetzung dieser Werthe die Gleichung 45 die Form an:

$$
\frac{1}{\mathrm{~B}}=\frac{1}{\mathrm{r}}\left\{\mathrm{n}[\mathrm{n} \mathrm{r}-(\mathrm{n}-1) \mathrm{d}]+\frac{(\mathrm{n}-1) \mathrm{d} \varrho}{\mathrm{Q}}\right\} \sin ^{2} \alpha .
$$

Ferner gelangt man, durch Einfiuhrung der gleichen Vernachlässigung in eine der Gleichungen 39, 40 oder 41, zu der Gleichung

$$
\sin \alpha=\frac{\mathrm{D}}{\mathrm{Q}} \sin \varepsilon,
$$

so dass also die gesuchte Beziehung zwischen Bildgite B und Incidenzwinkel $\varepsilon$ lautet:

$$
\frac{1}{\mathrm{~B}}=\frac{\mathrm{D}^{2}}{\mathrm{r} \mathrm{Q}^{2}}\left\{\mathrm{n}[\mathrm{nr}-(\mathrm{n}-1) \mathrm{d}]+\frac{(\mathrm{n}-1) \mathrm{d} \varrho}{\mathrm{Q}}\right\} \sin ^{2} \varepsilon .
$$

Es ergiebt sich also (für den angegebenen Winkelbereich) der Satz:

Bei einer gegebenen Linse ist die Bildgüte dem Quadrate des Sinus des Incidenzwinkels umgekehrt proportional.

Offenbar ist nun der Ausdruck $\mathrm{B} \sin ^{2} \varepsilon$ das natürliche vom Incidenzwinkel befreite Maass der Periscopie P, durch welches die periscopischen Eigenschaften verschiedener Linsen ohne Weiteres verglichen werden können. Wir setzen also 
Ueber Brechung b. schiefer Incidenz, m. besond. Berücksichtigung d. Auges. 375

und haben

$$
\mathrm{B} \sin ^{2} \varepsilon=\mathrm{P}
$$

$$
\frac{1}{\mathrm{P}}=\frac{\mathrm{D}^{2}}{\mathrm{r} \mathrm{Q}^{2}}\left\{\mathrm{n}[\mathrm{nr}-(\mathrm{n}-1) \mathrm{d}]+\frac{(\mathrm{n}-1) \mathrm{d} \varrho}{\mathrm{Q}}\right\}
$$

Es erweist sich nützlich in diese Gleichung die Haupt- oder Knotenpunctdistanz $\mathrm{E}$ der Linse einzuführen, welche ist

$$
\mathbf{E}=\frac{(\mathrm{n}-1) \mathrm{dD}}{\mathrm{Q}} \text {. }
$$

Soll dieselbe explicite in der Gleichung 49 enthalten sein, so muss eine der vier Constanten der Linse eliminirt werden. Wir wählen dazu den Brechungsindex n. Aus 50 ergiebt sich

$$
\mathbf{n}=\frac{\mathrm{d}(\mathrm{D}+\mathrm{E})}{\mathrm{D}(\mathrm{d}-\mathrm{E})}, \quad \mathbf{n}-1=\frac{\mathrm{E}(\mathrm{r}+\varrho)}{\mathrm{D}(\mathrm{d}-\mathrm{E})}, \quad \mathrm{Q}=\frac{\mathrm{d}(\mathrm{r}+\varrho)}{\mathrm{d}-\mathrm{E}}
$$

Setzt man diese Werthe in 49 ein, so entsteht nach einer Reihe von Umformungen der einfache Ausdruck

$$
\frac{1}{\mathrm{P}}=\frac{\mathrm{D}(\mathrm{D}+\mathrm{E})}{(\mathrm{r}+\varrho)^{2}}-\frac{\varrho}{\mathrm{r}} \frac{[\mathrm{D}(\mathrm{d}-\mathrm{E})+\mathrm{d}(\mathrm{r}+\varrho)]^{2}}{\mathrm{~d}^{2}(\mathrm{r}+\varrho)^{2}} .
$$

Von den beiden Gliedern dieses Ausdrucks ist das erste bei Systemen, welche noch wirkliche Linsengestalt haben, das bei weitem einflussreichere, und gewährt schon einen sehr annähernden Ausdruck für die. (reciproke) Periscopie. Es ist für $\mathrm{r}$ und $\varrho$ symmetrisch. Das zweite, kleinere Glied ist dagegen für $\mathrm{r}$ und $\varrho$ nicht symmetrisch. Da es für eine Linse stets negativ ist (denn $\mathrm{D}=\mathrm{r}+\varrho-\mathrm{d}$ ist positiv, und ebenso $\mathrm{d}-\mathrm{E}$ ), so folgt aus ihm, dass eine gegebene Convexlinse periscopischer ist, wenn ihre stärker gekrimmte Fläche dem Objecte zugekehrt wird. Indess werden wir nochmals auf diesen Schluss zurïckkommen, und iiberhaupt uns aus später anzugebenden Grïnden enthalten, aus Gleichung 52 weitere Schlïsse zu ziehen.

\section{Die Cardinalpuncte einer Linse für schiefen Strahlen- durchgang.}

Um zu weiteren Sätzen über den schiefen Durchgang zu gelangen, nehmen wir den optischen Mittelpunct der Linse als leuchtend an, und suchen seine von beiden Linsenflächen gelieferten Bilder. Bei axialen Strahlen sind diese Bilder bekanntlich die beiden Hauptpuncte. Die schiefen Strahlen geben für jeden gegebenen Incidenzwinkel je zwei, also vier Brennlinien, deren Orte 
in den Linien $\mathrm{LD}$ and $\mathrm{MD}^{\prime}$ der Figur 6 liegen müssen. Die Abstände des optischen Mittelpunctes von beiden Linsenflächen bei schiefer Incidenz sind die Linien $\mathrm{CA}$ und $\mathrm{CB}$, d. h. die beiden Abschnitte der Transversale $\mathrm{T}$, welche sich nach einem bekannten Satze verhalten wie die Krümmungsradien $\mathbf{r}$ und $\varrho$. Es ist also

$$
\mathrm{CA}=\frac{\mathrm{rT}}{\mathrm{r}+\varrho} \text { und } \mathrm{CB}=\frac{\varrho \mathrm{T}}{\mathrm{r}+\varrho} \text {. }
$$

Um die beiden von der vorderen Linsenfläche gelieferten Bilder des optischen Mittelpunctes zu finden, haben wir den Werth CA in die Gleichungen 1 und 2 für $f_{2}$ resp. $f_{1}$ einzuführen, und die Gleichungen für e zu lösen. Wir erhalten als Abstände der (virtuellen) Brennlinien, auf der Linie $L D$ von $A$ nach innen gemessen, die Werthe:

$$
\varphi_{2}=\frac{\mathrm{rT}}{\mathrm{n}(\mathrm{r}+\varrho)-\mathrm{AT}} \text { und } \varphi_{1}=\frac{\mathrm{NrT}}{\mathrm{n}(\mathrm{r}+\varrho) \cos ^{2} \alpha-\mathrm{AT}}
$$

und ebenso für die von der hinteren Fläche gelieferten virtuellen Bilder des optischen Mittelpunetes die Abstände (von B aus nach $\mathrm{D}^{\prime}$ hin gemessen):

$$
\psi_{2}=\frac{\varrho \mathrm{T}}{\mathrm{n}(\mathrm{r}+\varrho)-\mathrm{AT}} \text { und } \quad \psi_{1}=\frac{\mathrm{N} \varrho \mathrm{T}}{\mathrm{n}(\mathrm{r}+\varrho) \cos ^{2} \alpha-\mathrm{AT}}
$$

In dem Werthe von $\psi_{2}$ erkennen wir sofort wieder die schon oben Seite 373 bestimmte und mit $\mathrm{S}$ bezeichnete Strecke BD', and ebenso ergiebt sich aus der Betrachtung des Dreiecks HAD, dass $\varphi_{2}$ nichts anderes ist als die Länge $\mathrm{AD}$. $\mathrm{Da} \mathrm{D}$ und $\mathrm{D}^{\prime}$ diejenigen Puncte sind, welche im 1. Theil als die "Directionspuncte" bezeichnet wurden, so ergiebt sich:

Die schiefwinkligen zweiten Bilder ${ }^{1}$ ) des optischen Mittelpuncts sind die Directionspuncte (von denen schon erwähnt ist, dass sie bei abnehmendem Incidenzwinkel schliesslich in die Knoten- oder Hauptpuncte iibergehen).

Die Directionspuncte sind also auch untereinander conjugirt, der eine das zweite Bild des andern.

Die ersten Bilder $\mathrm{E}$ und $\mathrm{E}^{\prime}$ des optischen Mittelpuncts, welche

1) Es wäre besser gewesen, wenn von Anfang an die Indices 1 und 2 für beide Brennlinien vertauscht worden wären; denn die jetzt mit dem Index 2 versehenen stellen durchweg den einfacheren Fall dar. Ich habe jedoch die von mir angewandte Bezeichnung schon in der Literatur vorgefunden, und eine Aenderung würde jetzt Verwirrung anrichten. 
von A und B um die Strecken $\varphi_{1}$ resp. $\psi_{1}$ entfernt sind, liegen dagegen nicht in der Axe, sondern in den Linien $\mathrm{AD}$ und $\mathrm{BD}^{\prime}$, von den Directionspuncten um die Strecke $\mathrm{DE}=\varphi_{2}-\varphi_{1}$, resp. $\mathrm{D}^{\prime} \mathrm{E}^{\prime}=\psi_{2}-\psi_{1}$ entfernt. Ich werde sie als "Seitenpuncte" bezeichnen; also

die schiefwinkligen ersten Bilder des optischen Mittelpuncts sind die Seitenpuncte,

welche ebenfalls unter einander conjugirt sind.

Beim Incidenzwinkel Null fallen Knoten-, Directions- und Seitenpuncte zusammen. Mit zunehmendem Incidenzwinkel rücken die Directions- und Seitenpuncte von den Knotenpuncten fort, und zwar erstere in der Axe, letztere in einer eigenthümlichen Curve, indem sie sich sowohl von der Mitte als von der Axe der Linse entfernen.

Weitere wichtige Beziehungen erhält man, wenn man die Entfernungen der Brennpuncte der Linse nicht von der Axe, sondern von den gleichnamigen inneren Cardinalpuncten aus bemisst, also die Werthe $\mathrm{F}_{2}+\psi_{2}, \mathrm{~F}_{1}+\psi_{1}, \mathrm{f}_{2}+\psi_{2}, \mathrm{f}_{1}+\psi_{1}, \mathrm{e}_{2}+\varphi_{2}, \mathrm{e}_{1}+\phi_{1}$ einführt. Man erhält dann aus den Gleichungen 26, 271), 35, 36, 53, 54 die folgenden, deren Ableitung ich dem Leser überlasse:

$$
\begin{aligned}
& \frac{1}{\mathrm{e}_{2}+\varphi_{2}}+\frac{1}{\mathrm{f}_{2}+\psi_{2}}=\frac{1}{\mathrm{~F}_{2}+\psi_{2}} \\
& \frac{1}{\mathrm{e}_{1}+\varphi_{1}}+\frac{1}{\mathrm{f}_{1}+\psi_{1}}=\frac{1}{\mathrm{~F}_{1}+\psi_{1}} .
\end{aligned}
$$

Diese Gleichungen gelten in voller Strenge. Bezeichnet man ferner mit $E_{2}$ und $E_{1}$ die vorderen Hauptbrennweiten der Linse für schiefe Incidenz, so ergiebt sich aus denselben Urgleichungen:

$$
\mathrm{E}_{2}+\varphi_{2}=\mathrm{F}_{2}+\psi_{2} \text { und } \mathrm{E}_{1}+\varphi_{1}=\mathrm{F}_{1}+\psi_{1},
$$

so dass man die folgenden, ebenfalls völlig strengen Gleichungen erhält:

$$
\begin{aligned}
& \frac{\mathrm{E}_{2}+\varphi_{2}}{\mathrm{e}_{2}+\varphi_{2}}+\frac{\mathrm{F}_{2}+\psi_{2}}{\mathrm{f}_{2}+\psi_{2}}=1 \\
& \frac{\mathrm{E}_{1}+\varphi_{1}}{\mathrm{e}_{1}+\varphi_{1}}+\frac{F_{1}+\psi_{1}}{f_{1}+\psi_{1}}=1
\end{aligned}
$$

1) Diese beiden Gleichungen sind zuvor für Mitteldurchgang umzugestalten, indem $\mathrm{M}=\mathrm{N}, \mathrm{B}=\mathrm{A}, \beta=\alpha$ gesetzt wird. 
welche den bekannten Gleichungen für centralen Durchgang völlig analog und zur Berechnung der schiefwinkligen Bilder endlich entfernter $O b j e c t e$ sehr wichtig sind. Für die dünne Linse gehen sie in die schon angeführten Gleichungen 11 und 12 über.

Man ersieht hieraus, dass die Directions- and Seitenpuncte zu den schiefwinkligen Brennpuncten genau in gleicher Beziehung: stehen, wie bei normalem Durchgang die Haupt- oder Knotenpuncte, so dass wir volles Recht haben, sie als schiefwinklige Cardinalpuncte der Linse zu bezeichnen.

Von grossem Interesse ist es noch, die gegenseitigen Distanzen dieser Puncte zu berechnen.

Für die Distanz der Directionspuncte $\mathrm{DD}^{\prime}=\Delta_{2}$ haben wir schon die Gleichung 42 berechnet, wonach

$$
\Delta_{2}=\frac{\mathrm{D} \cdot \mathrm{AT}}{\mathrm{n}(\mathrm{r}+\varrho)-\mathrm{AT}} .
$$

Bis auf Grössen dritter Ordnung genan erhalten wir hieraus

$$
\Delta_{2}=\frac{(\mathrm{n}-1) \mathrm{dD}}{\mathrm{Q}}+\frac{(\mathrm{n}-1) \mathrm{dD}}{2 \mathrm{Q}} \cdot \mathrm{n}(\mathrm{r}+\varrho)\left(\frac{1}{\mathrm{D}}+\frac{1}{\mathrm{Q}}\right) \sin ^{2} \alpha,
$$

und da die Distanz der Haupt- oder Knotenpuncte

so ist

$$
\mathrm{E}=\frac{(\mathrm{n}-1) \mathrm{dD}}{\mathrm{Q}}
$$

$$
\Delta_{2}=\mathrm{E}+\frac{\mathrm{E}}{2} \mathrm{n}(\mathrm{r}+\varrho)\left(\frac{1}{\mathrm{D}}+\frac{1}{\mathrm{Q}}\right) \sin ^{2} \alpha .
$$

Bezeichnen wir weiter mit $\Lambda_{1}=\mathrm{EE}^{\prime}$ die Distanz beider Seitenpuncte, so finden wir dieselbe aus dem Dreieck NEE' (Fig. 6), in welchem $\mathrm{NE}^{\prime}=\mathrm{DD}^{\prime}=A_{2}, \mathrm{NE}=\varphi_{2}-\varphi_{1}+\psi_{2}-\psi_{1}$ und Winkel $\mathrm{ENE}^{4}=180^{\circ}-\varepsilon . \quad$ Es ist also

(61) $\Delta_{1}^{2}=\Delta_{2}^{2}+\left(\varphi_{2}-\varphi_{1}+\psi_{2}-\psi_{1}\right)^{2}+2 \Delta_{2}\left(\varphi_{2}-\varphi_{1}+\psi_{2}-\psi_{1}\right) \cos \varepsilon$. Eine genaue Berechnung ergiebt einen höchst complicirten Ausdruck. Beschränken wir uns aber auf den schon früher gewählten Gültigkeitsbereich, so haben wir

$$
\begin{gathered}
\Delta_{2}^{2}=\mathrm{E}^{2}+\mathrm{E}^{2} \mathrm{n}(\mathrm{r}+\varrho)\left(\frac{1}{\mathrm{D}}+\frac{1}{\mathrm{Q}}\right) \sin ^{2} \alpha \text { (aus 60), } \\
\varphi_{2}-\varphi_{1}+\psi_{2}-\psi_{1}=\mathrm{n}(\mathrm{r}+\varrho) \mathrm{d} \frac{\mathrm{nQ}-(\mathrm{r}+\varrho)}{\mathrm{Q}^{2}} \sin ^{2} \alpha(\text { aus } 53,54) ;
\end{gathered}
$$

da ferner

$$
\mathrm{nQ}-(\mathrm{r}+\varrho)=(\mathrm{n}-1)(\mathrm{nD}+\mathrm{r}+\varrho)=(\mathrm{n}-1)(\mathrm{D}+\mathrm{Q}),
$$

so können wir schreiben 
Ueber Brechung b. schiefer Incidenz, m. besond. Berücksichtigung d. Auges. 379

$$
\begin{gathered}
\varphi_{2}-\varphi_{1}+\psi_{2}-\psi_{1}=\mathrm{n}(\mathrm{n}-1)(\mathrm{r}+\varrho) \mathrm{d} \frac{\mathrm{D}+\mathrm{Q}}{\mathrm{Q}^{2}} \sin ^{2} \alpha \\
=\mathrm{n}(\mathrm{r}+\varrho) \mathrm{E}\left(\frac{1}{\mathrm{D}}+\frac{1}{\mathrm{Q}}\right) \sin ^{2} \alpha .
\end{gathered}
$$

Endlich ist nach Gleichung 42

$$
A_{2} \cos \varepsilon=\sqrt{\mathcal{A}_{2}^{2}-\mathrm{A}^{2} \mathrm{~T}^{2} \sin ^{2} \alpha}
$$

und da dieser Werth in 61 nur als Factor eines schon mit $\sin ^{2} \alpha$ multiplicirten Gliedes vorkommt, so ist in unserm Falle dafür zu setzen

$$
(\mathrm{n}-1) \mathrm{d} \sqrt{\frac{\mathrm{D}^{2}}{\mathrm{Q}^{2}}-\sin ^{2} \alpha}, \quad \text { oder } \frac{(\mathrm{n}-1) \mathrm{dD}}{\mathrm{Q}}=\mathrm{E}
$$

Wir erhalten somit aus 61 , da das zweite Glied der rechten Seite wegen des Factors $\sin ^{4} \alpha$ ganz ausfällt,

$A_{1}^{2}=\mathrm{E}^{2}+\mathrm{E}^{2} \mathrm{n}(\mathrm{r}+\varrho)\left(\frac{1}{\mathrm{D}}+\frac{1}{\mathrm{Q}}\right) \sin ^{2} \alpha+2 \mathrm{E}^{2} \mathrm{n}(\mathrm{r}+\varrho)\left(\frac{1}{\mathrm{D}}+\frac{1}{\mathrm{Q}}\right) \sin ^{2} \alpha$, so dass

$$
\Delta_{1}=\mathrm{E}+\frac{3}{2} \mathrm{En}(\mathrm{r}+\varrho)\left(\frac{1}{\mathrm{D}}+\frac{1}{\mathrm{Q}}\right) \sin ^{2} \alpha_{\circ}
$$

Aus 60 und 62 folgt also:

(63) $\left.\Delta_{1}-\Delta_{2}=\mathrm{E} \cdot \mathrm{n}(\mathrm{r}+\varrho)\left(\frac{1}{\mathrm{D}}+\frac{1}{\mathrm{Q}}\right) \sin ^{2} \alpha=\left(\varphi_{2}+\psi_{2}\right)-\left(\varphi_{1}+\psi_{1}\right)^{1}\right)$ und hieraus die merkwürdige, natürlich nur für den angegebenen Winkelbereich gültige Beziehung

$$
3 A_{2}-\Delta_{1}=2 \mathrm{E} \text {. }
$$

Durch die Distanz der Haupt- und der Directionspuncte ist also jedesmal auch die der Seitenpuncte gegeben, ohne dass die unmittelbaren Constanten der Linse in Betracht kommen.

\section{Modificirte Bemessung der Bildgüte und der Periscopie.}

Ueberraschend einfache Ausdrücke erhält man, wenn man auch in den oben bezeichneten Werthen für $\mathrm{B}$ und $\mathrm{P}$ statt der Brennweiten $\mathrm{F}_{2}, \mathrm{~F}_{1}$, die Längen $\mathrm{F}_{2}+\psi_{2}, \mathrm{~F}_{1}+\psi_{1}$ einführt, $\mathrm{d}$. h. die Bilddistanzen von den zugehörigen inneren Cardinalpuncten aus misst. Wir setzen also

1) Die Gleichung $\Delta_{1}=\Delta_{2}+\left(\varphi_{2}+\psi_{2}\right)-\left(\varphi_{1}+\psi_{1}\right)$ erhält man kürzer direct durch Betrachtung des Dreiecks ENE' unter Berücksichtigung der angenommenen Kleinheit des Winkels $\varepsilon$. 
(65)

$$
\frac{1}{\mathrm{~B}^{\prime}}=\frac{\left(\mathrm{F}_{2}+\psi_{2}\right)-\left(\mathrm{F}_{1}+\psi_{1}\right)}{\mathrm{F}_{2}+\psi_{2}}, \quad \text { und } \quad \mathrm{P}^{\prime}=\mathrm{B}^{\prime} \sin ^{2} \varepsilon
$$

zunächst ohne Prüfung der physicalischen Bedentung dieses Ausdrucks.

Aus den bisherigen Gleichungen ergiebt sich

$$
\begin{gathered}
\mathrm{F}_{2}+\psi_{2}=\frac{1}{\mathrm{~A}} \cdot \frac{\mathrm{nr} \varrho}{\mathrm{n}(\mathrm{r}+\varrho)-\mathrm{AT}} \\
\mathrm{F}_{1}+\psi_{1}=\frac{\mathrm{N}}{\mathrm{A}} \cdot \frac{\mathrm{nr} \varrho \cos ^{2} \alpha}{\mathrm{n}(\mathrm{r}+\varrho) \cos ^{2} \alpha-\mathrm{AT}} .
\end{gathered}
$$

Führt man auch hier Näherungswerthe ein, so ist, wenn man berüeksichtigt, dass

$$
\frac{\mathrm{nr} \varrho}{(\mathrm{n}-1) \mathrm{Q}}=\mathrm{F} \text { und } \mathrm{n}+\frac{\mathrm{r}+\varrho}{\mathrm{D}}=\frac{\mathrm{Q}+\mathrm{D}}{\mathrm{D}}
$$

68) $\quad \mathrm{F}_{2}+\psi_{2}=\mathrm{F}-\frac{\mathrm{F}}{2}\left(\mathrm{n}-(\mathrm{n}-1)\left(\frac{1}{\bar{D}}+\frac{1}{\mathrm{Q}}\right) \mathrm{d}\right) \sin ^{2} \alpha$,

(69) $\mathrm{F}_{1}+\psi_{1}=\mathrm{F}_{2}+\psi_{2}-\mathrm{F}\left(\mathrm{n}^{2}-\frac{(\mathrm{n}-1) \mathrm{d}}{\mathrm{Q}}\right) \sin ^{2} \alpha$,

so dass

(70) $\frac{1}{\mathrm{~B}^{\prime}}=\left(\mathrm{n}^{2}-\frac{(\mathrm{n}-1) \mathrm{d}}{\mathrm{Q}}\right) \sin ^{2} \alpha=\frac{\mathrm{D}^{2}}{\mathrm{Q}^{2}}\left(\mathrm{n}^{2}-\frac{(\mathrm{n}-1) \mathrm{d}}{\mathrm{Q}}\right) \sin ^{2} \varepsilon$

und

$$
\frac{1}{\mathrm{P}^{2}}=\frac{\mathrm{D}^{2}}{\mathrm{Q}^{2}}\left(\mathrm{n}^{2}-\frac{(\mathrm{n}-1) \mathrm{d}}{\mathrm{Q}}\right)
$$

Bildet man weiter durch Division der Gleichungen 60 und 63 den Ausdruck

$$
\frac{\Delta_{1}-\Delta_{2}}{\Delta_{2}}\left(=2 \cdot \frac{\Delta_{2}-\mathrm{E}}{\mathrm{E}}\right)=\mathrm{n}(\mathrm{r}+\varrho)\left(\frac{1}{\mathrm{D}}+\frac{1}{\mathrm{Q}}\right) \sin ^{2} \alpha,
$$

so erhält man dureh Umformung, da

$$
\begin{aligned}
& \begin{aligned}
\mathrm{n}(\mathrm{r}+\varrho) \frac{\mathrm{D}+\mathrm{Q}}{\mathrm{DQ}} & =\frac{\mathrm{n}(\mathrm{r}+\varrho)}{\mathrm{n}-1} \cdot \frac{\mathrm{nQ}-(\mathrm{r}+\varrho)}{\mathrm{DQ}}=\frac{\mathrm{r}+\varrho}{(\mathrm{n}-1) \mathrm{D}}\left(\mathrm{n}^{2}-\frac{\mathrm{n}(\mathrm{r}+\varrho)}{\mathrm{Q}}\right) \\
& =\frac{\mathrm{d}-\mathrm{E}}{\mathrm{E}}\left(\mathrm{n}^{2}-\frac{\mathrm{n}(\mathrm{r}+\varrho)}{\mathrm{Q}}\right),
\end{aligned} \\
& \text { (72) } \\
& \quad 2 \cdot \frac{\Delta_{2}-\mathrm{E}}{\mathrm{d}-\mathrm{E}}=\left(\mathrm{n}^{2}-\frac{\mathrm{n}(\mathrm{r}+\varrho)}{\mathrm{Q}}\right) \sin ^{2} \alpha,
\end{aligned}
$$

und subtrahirt man endlich die Gleichung 72 von 70 , so erhält man die merkwïrdige Gleichung

$$
\frac{1}{\mathrm{~B}^{\prime}}=2 \frac{\Delta_{2}-\mathrm{E}}{\mathrm{d}-\overline{\mathrm{E}}}+\sin ^{2} \alpha \text {. }
$$

Ferner kann man noch aus den Gleichungen 70 und $73 \sin ^{2} \alpha$ eliminiren, und erhält so 
Ueber Brechung b. schiefer Incidenz, m. besond. Berücksichtigung d. Auges. 381

(74) $\frac{1}{\mathrm{~B}^{\prime}}=2 \frac{A_{2}-\mathrm{E}}{\mathrm{d}-\mathrm{E}} \cdot \frac{\mathrm{n}^{2} \mathrm{Q}-(\mathrm{n}-1) \mathrm{d}}{\mathrm{n}^{2} \mathrm{Q}-\mathrm{n}(\mathrm{r}+\varrho)}=2 \frac{A_{2}-\mathrm{E}}{\mathrm{d}-\mathrm{E}}\left(1+\frac{\mathrm{Q}}{\mathrm{n}^{2} \mathrm{Q}-\mathrm{n}(\mathrm{r}+\varrho)}\right)$.

Diese Gleichung enthält den Incidenzwinkel nur noch in dem Werth $\Delta_{2}$. Für Umformungen der Gleichungen $72-74$ ist zu beachten, dass

$$
2 \frac{\Delta_{2}-\mathrm{E}}{\mathrm{d}-\mathrm{E}}=\frac{\Delta_{1}-\Delta_{2}}{\mathrm{~d}-\mathrm{E}}=\frac{\left(\varphi_{2}+\psi_{2}\right)-\left(\varphi_{1}+\psi_{1}\right)}{\varphi_{2}+\psi_{2}} .
$$

Endlich kann man aus 74 noch $n$ mittels der Gleichungen 51 herausschaffen, und erhält dann

(75) $\quad \frac{1}{\bar{B}^{\prime}}=\frac{2\left(A_{2}-E\right)}{\mathrm{E}(\mathrm{d}-\mathrm{E})(\mathrm{D}+\mathrm{E})} \cdot \frac{\mathrm{d}^{2}(\mathrm{D}+\mathrm{E})^{2}-\mathrm{DE}(\mathrm{d}-\mathrm{E})^{2}}{\mathrm{~d}^{2}-\mathrm{DE}+2 \mathrm{dD}}$

und ebenso kann man aus $74 \mathrm{r}$, $\varrho$ and $Q$ berausschaffen (mittels 51) und erhält

$$
\frac{1}{\mathrm{~B}^{\prime}}=2 \frac{\Delta_{2}-\mathrm{E}}{\mathrm{d}-\mathrm{E}}\left(1+\frac{\mathrm{d}}{\mathrm{n}[\mathrm{E}+(\mathrm{n}-1) \mathrm{d}]}\right)
$$

Untersuchen wir nunmehr die physicalische Bedeutung des Werthes $B^{\prime}$, für welchen wir so merkwürdige und einfache Beziehungen gefunden haben. Früher maassen wir die Astigmasie des Bildes nach der Länge der Brennstrecke, bezogen auf die Grösse des Bildes; dies war der Werth $\frac{1}{\mathrm{~B}} ; \frac{1}{\mathrm{~B}^{\prime}}$ dagegen ist die Differenz der beiden Bildgrössen, bezogen anf ihre absolute Grösse; denn die Grössen der Bilder sind, wie man leieht findet, den Werthen $\mathrm{F}_{2}+\psi_{2}$, resp. $\mathrm{F}_{1}+\psi_{1}$, proportional; im Nenner aber ist es, bei dem gewählten Näherungsgrade, einerlei, ob wir $F_{1}+\psi_{1}$ oder $\mathrm{F}_{2}+\psi_{2}$ oder auch $\mathrm{F}$ setzen.

Offenbar lässt sich auch B', ebensogut wie B, als ein Maass der Bildgüte, und $\mathrm{P}^{\prime}$ so gut wie $\mathrm{P}$ als ein Maass der Periscopie betrachten. Die neuen Maasse aber unterscheiden sich von den frïheren dadurch, dass in ihnen $\mathrm{r}$ und $\varrho$ durchweg symmetrisch vorkommen, so dass es gleichgültig ist, welche Seite der Linse dem Objecte zugekehrt wird. Für die dünne Linse geben beide Messweisen gleiche Resultate.

Der Grösse nach unterscheiden sich die Werthe $\frac{1}{\mathrm{~B}}$ und $\frac{1}{\mathrm{~B}^{\prime}}$ dadurch, dass ersterer, bei gleichem Nenner, einen um den Betrag $\psi_{2}-\psi_{1}$ grösseren Zähler hat.

Das neue Maass der Bildgüte, B', erscheint mir als das richtigere, weil eine Correctur des astigmatisehen Bildes vor Allem beide Bilder gleich gross zu machen hätte; wir kommen hierauf 
im nächsten Paragraphen zuriick. Die bedeutend einfacheren Ausdrücke scheinen dies zu bestätigen.

Zusatz. Die äusseren Brennpuncte beider Fläehen einer Linse sind bekanntlich unter einander conjugirt; das von dem einen ausgehende Strahlenbündel geht parallelstrahlig durch die Linse und vereinigt sich nach der zweiten Brechung im anderen. Dasselbe gilt natürlich auch für schiefen Durehgang; wir gewinnen so zwei weitere Paare conjugirter Puncte, das eine mit dem Index 1, das andere mit dem Index 2 versehen. Um die Lage derselben für den Fall des Durchgangs durch den optisehen Mittelpunct zu finden, hat man einfach in den Gleichungen $1 \mathrm{f}_{\mathbf{2}}$ resp. $\mathrm{f}_{\mathbf{1}}=\infty \mathrm{zu}$ setzen und für e zu lösen; für die zweite Fläche ist $\varrho$ statt $r$ zu nehmen. Man erhält für die erste Fläche

$$
\mathrm{G}_{2}=\frac{\mathrm{r}}{\mathrm{A}}, \quad \mathrm{G}_{1}=\frac{\mathrm{r} \cos ^{2} \varphi}{\mathrm{A}}=\frac{\mathrm{Nr}}{\mathrm{A}} .
$$

Bemisst man die Abstände vom zugehörigen Directions- resp. Seitenpuncte aus, so erhält man

$$
\mathrm{G}_{2}+\varphi_{2}=\frac{\mathrm{nr}(\mathrm{r}+\varrho)}{\mathrm{n}(\mathrm{r}+\varrho)-\mathrm{AT}} ; \quad \mathrm{G}_{1}+\varphi_{1}=\frac{\mathrm{nNr}(\mathrm{r}+\varrho) \cos ^{2} a}{\mathrm{n}(\mathrm{r}+\varrho) \cos ^{2} \alpha-A \mathrm{~T}}
$$

und ferner ergiebt sich leicht

$$
\frac{\left(\mathrm{G}_{2}+\varphi_{2}\right)-\left(\mathrm{G}_{1}+\varphi_{1}\right)}{\mathrm{G}_{2}+\varphi_{2}}=\frac{1}{\mathrm{~B}^{\prime}},
$$

so dass man nur die Lage der äusseren schiefen Brennpuncte der einen Linsenfläche zu kennen braucht, um das Maass der Bildgüte und Periscopie zu gewinnen.

Bezeichnet man ferner als Seitenverschiebung $\Sigma$ den Verticalabstand des einfallenden und gebrochenen Bündels (DP in Fig. 6), so ergiebt sich

$$
\Sigma=A_{2} \sin \varepsilon
$$

und bis auf Grössen dritter Ordnung genau:

$$
\Sigma=\mathbf{E} \sin \varepsilon \text {. }
$$

\section{Bestimmung der corrigirenden Cylindergläser.}

Die Astigmasie eines Bildes lässt sich auch nach der optischen Kraft desjenigen Cylinderglases bemessen, welches das eine Bild an den Ort des anderen verlegt. Nach einem bekannten Princip ist die optische Kraft, oder der reciproke Werth der Brennweite eines solchen Glases gleich der Differenz der reciproken Werthe der beiden gleich zu machenden Brennweiten, wenn beide von demselben Puncte aus zählen und an diesen Punct das corrigirende Glas verlegt wird. 
Ueber Brechung b. schiefer Incidenz, m. besond. Berücksichtigung d. Auges. 383

Nennen wir die Brennweite des gesuchten Cylinderglases D, und versetzen wir dasselbe, senkrecht zum Leitstrahl, an den Ort des zweiten Directionspunets, so ergiebt sich

$$
\frac{1}{\Phi}=\frac{1}{\mathrm{~F}_{1}+\psi_{2}}-\frac{1}{\mathrm{~F}_{2}+\psi_{2}}
$$

und man erhält

$$
\frac{1}{\Phi}=\frac{\mathrm{n}-1}{\mathrm{n}} \cdot \frac{\mathrm{D}^{2}}{\mathrm{r}^{2} \varrho \mathrm{Q}}\left\{\mathrm{n}[\mathrm{nr}-(\mathrm{n}-1) \mathrm{d}]+\frac{(\mathrm{n}-1) \mathrm{d} \varrho}{\mathrm{Q}}\right\} \sin ^{2} \varepsilon
$$

und aus der Vergleichung dieses Ausdrucks mit 48 findet man

$$
\frac{1}{\Phi}=\frac{1}{B} \frac{(\mathrm{n}-1) \mathrm{Q}}{\mathrm{nr} \varrho}=\frac{1}{\mathrm{~B}} \cdot \frac{1}{\mathrm{~F}}
$$

so dass also

und

$$
\begin{gathered}
\Phi=\mathrm{B} . \mathrm{F} \\
\mathrm{P}=\frac{\Phi}{\mathrm{F}} \sin ^{2} \varepsilon .
\end{gathered}
$$

Setzen wir aber, vorbehaltlich physicalischer Deutung des Ausdrucks,

so ergiebt sich

$$
\frac{1}{\Phi^{i}}=\frac{1}{\mathrm{~F}_{1}+\psi_{1}}-\frac{1}{\mathrm{~F}_{2}+\psi_{2}}
$$

$$
\frac{1}{D^{\prime}}=\frac{\mathrm{n}-1}{\mathrm{nr} \varrho} \cdot \frac{D^{2}}{\mathrm{Q}}\left(\mathrm{n}^{2}-\frac{(\mathrm{n}-1) \mathrm{d}}{\mathrm{Q}}\right) \sin ^{2} \varepsilon,
$$

oder

und

$$
\begin{gathered}
\frac{1}{\Phi^{\prime}}=\frac{1}{\mathrm{~B}^{\prime}} \cdot \frac{1}{\mathrm{~F}} \quad \text { oder } \quad \Phi^{\prime}=\mathrm{B}^{\prime} \cdot \mathrm{F}, \\
\mathrm{P}^{\prime}=\frac{\Phi^{\prime}}{\mathrm{F}} \sin ^{2} \varepsilon .
\end{gathered}
$$

Wie man sieht, steht der Werth $\Phi^{\prime}$ zu $\Phi$ in genau derselben Beziehung wie $B^{\prime}$ zu B. Die physicalische Bedeutung ron $\Phi^{\prime}$ ist aber leicht übersehbar; es ist nämlich die Brennweite eines $\mathrm{Cy}$ linderglases, welches das Bild homocentrisch machen würde, wenn die Brennweiten beider Brennlinien von demselben Puncte ausgingen, was in Wirklichkeit nicht der Fall ist. Eine wahre Correctur des Bildes wie es wirklich ist, ist ïberhaupt nicht durch eine einfache Cylinderlinse möglich, sondern man muss die Verschiebung der einen Brennweite gegen die andere (bis Seitenpunet auf Directionspunct fällt) fingiren, um zu einer corrigirenden Linse zu gelangen, deren Brennweite dann $\omega^{\prime}$ ist. Man sieht hieraus, dass in der That die Werthe $B^{\prime}, P^{\prime}, \Phi^{\prime}$ theoretisch richtigere Maasse sind, als die entsprechenden ohne Index. 
Die Astigmasie durch die corrigirende (fingirte) Cylinderlinse zu messen, empfiehlt sich besonders deshalb, weil dieses Maass zugleich für unendlich und für endlich entfernte Objecte gilt, also allgemeiner ist als $\mathrm{B}^{\prime}$ und $\mathrm{P}^{\prime}$. Es ist nämlich nach Gleichung 55 und 56 für ein endlich entferntes Object vom Abstand $\mathrm{e}_{1}=\mathrm{e}_{2}=\mathrm{e}$ (vgl. I. Theil S. 448), sobald dieser so gross ist, dass die Differenz von $\varphi_{1}$ und $\varphi_{2}$ dagegen versehwindet,

$$
\frac{1}{\mathrm{f}_{1}+\psi_{1}}-\frac{1}{\mathrm{f}_{2}+\psi_{2}}=\frac{1}{\mathrm{~F}_{1}+\psi_{1}}-\frac{1}{\mathrm{~F}_{2}+\psi_{2}}=\frac{1}{\Phi^{\prime}} .
$$

10. Vergleichung der Periscopie verschiedener Linsen.

Um die Periscopie versehiedener Linsen bequem zu vergleichen, benutzen wir die Gleichung 71:

$$
\frac{1}{\mathrm{P}^{\prime}}=\frac{\mathrm{D}^{2}}{\mathrm{Q}^{2}}\left(\mathrm{n}^{2}-\frac{(\mathrm{n}-1) \mathrm{d}}{\mathrm{Q}}\right) .
$$

1. Unendlich dünne Linsen. Hier ist $d=0, D=r+\varrho$, $\mathrm{Q}=\mathrm{n}(\mathrm{r}+\varrho)$. Es ergiebt sich also $\mathrm{P}^{\prime}=1$. Die Periscopie unendlich dünner Linsen ist also gleich 1 , was schon aus dem 5. Paragraphen hervorgeht, da dort $\frac{1}{\mathrm{~B}^{\prime}}=\sin ^{2} \varphi=\sin ^{2} \varepsilon$ sich ergab, also $\mathrm{P}^{\prime}=\mathrm{B}^{\prime} \sin ^{2} \varepsilon=1$.

2. Kugellinsen, und überhaupt Linsen, deren Dicke gleich der Summe beider Krümmungsradien ist. Hier ist $\mathrm{D}=0$, also $\mathrm{P}^{\prime}=\infty$; solche Linsen sind unendlich periscopisch, was schon die Anschauung lehrt, da jedes durch den optischen Mittelpunct gehende Strahlenbündel durch beide Flächen senkrecht hindurchgeht.

3. Andere Fälle. Für solche ist es zweckmässig, die Gleichung 71 etwas umzuformen. Es ist nämlich

$$
\begin{aligned}
\mathrm{n}^{2}-\frac{(\mathrm{n}-1) \mathrm{d}}{\mathrm{Q}} & =\mathrm{n}^{2}+\frac{\mathrm{Q}-\mathrm{n}(\mathrm{r}+\varrho)}{\mathrm{Q}}=\mathrm{n}^{2}+1-\frac{\mathrm{n}(\mathrm{r}+\varrho)}{\mathrm{Q}} \\
& \left.=\mathrm{n}^{2}+1-\mathrm{n}\left(1-\frac{\mathrm{E}}{\mathrm{d}}\right) \mathrm{s}\right) ;
\end{aligned}
$$

ferner nach Gleichung 50:

$$
\frac{\mathrm{D}}{\mathrm{Q}}=\frac{\mathrm{E}}{(\mathrm{n}-1) \mathrm{d}},
$$

also

1) Vgl. Gleichung 51 . 
Ueber Brechung b. schiefer Incidenz, m. besond. Berücksichtigung d. Auges. 385

$$
\begin{aligned}
\frac{1}{\mathbf{P}^{2}} & =\frac{\mathrm{E}^{2}}{(\mathrm{n}-1)^{2} \mathrm{~d}^{2}}\left(\mathrm{n}^{2}-\mathrm{n}+1+\mathrm{n} \frac{\mathrm{E}}{\mathrm{d}}\right) \\
& =\frac{\mathrm{E}^{2}}{\mathrm{~d}^{2}}\left[1+\frac{\mathrm{n}}{(\mathrm{n}-1)^{2}}\left(1+\frac{\mathrm{E}}{\mathrm{d}}\right)\right] .
\end{aligned}
$$

Aus dieser Gleichung, in weleher die Krümmungsradien nicht vorkommen, folgt, dass von allen Linsen mit gegebener Dicke und gegebenem Brechungsindex diejenige am meisten periscopisch ist, welche die kleinste Hauptpunctdistanz besitzt. Die unendlich duinne und die Kugellinse haben beide $\mathrm{E}=0$, indess ist für erstere auch $\mathrm{d}=0$, also $\frac{1}{\mathrm{P}^{\prime}}=\frac{0}{0}$; der wahre Werth $(=1)$ ist aber schon oben ermittelt. Die anendlich dünne Linse ist unter allen Linsen gleicher Brennweite die am wenigsten periscopische.

Führen wir statt des Brechungsindex die Krümmungsradien der Linse ein, wozu die Gleichungen 51 dienen, so erhalten wir

$$
\frac{1}{\mathrm{P}^{\prime}}=\frac{\mathrm{E}^{2}}{\mathrm{~d}^{2}}+\frac{\mathrm{D}(\mathrm{D}+\mathrm{E})(\mathrm{d}-\mathrm{E})}{\mathrm{d}(\mathrm{r}+\varrho)^{2}}\left(1+\frac{\mathrm{E}}{\mathrm{d}}\right)
$$

und mit Einsetzung des Werthes von D, sowie nach mehreren Umformungen

$$
\frac{1}{\mathrm{P}^{\prime}}=1-\frac{\mathrm{d}^{2}-\mathrm{E}^{2}}{\mathrm{~d}(\mathrm{r}+\varrho)}\left(2-\frac{\mathrm{d}-\mathrm{E}}{\mathrm{r}+\varrho}-\frac{\mathrm{E}}{\mathrm{d}}\right) .
$$

Hieraus folgt, dass unter allen Linsen von gegebener Gestalt $(\mathrm{r}, \varrho, \mathrm{d})$ diejenige am meisten periscopisch ist, welche die kleinste Hauptpunctdistanz besitzt.

Es muss ausdricklich bemerkt werden, dass dieser Satz nur für eine homogene Linse bewiesen ist, also nicht etwa ohne Weiteres auf die Krystalllinse übertragen werden darf, obgleich diese, wie eine einfache Betrachtung lehrt'), eine viel kleinere Haupt-

1) Helmboltz hat nachgewiesen (physiologische Optik S. 75), dass die Krystalllinse vermöge ihres eigenthümlichen geschichteten Baues eine kürzere Hauptpunotdistanz hat, als eine homogene Linse von' gleicher äusserer Gestalt und vom Brechungsvermögen des Kerns. Hieraus folgt aber a fortiori dass die Hauptpunctdistanz der Krystalllinse kleiner ist als die einer homogenen Linse von gleicher Gestalt und gleicher Brennweite; denn bekanntlich ist der Index der letzteren Linse (der sogenannte Totalindex der Krystalllinse) grösser als der des Kerns der Krystalllinse, ihre Hauptpunctdistanz ist also noch grösser als die der homogenen Linse vom Kernindex, da nach Gleichung 50 die Hauptpunctdistanz mit dem Index zunimmt. Bei gegebener Brennweite und gegebener äusserer Gestalt wird also die Hauptpunctdistanz durch diem jenige Schichtungsart, welche die Krystalllinse besitzt, beträ.chtlich vermindert. 
punctdistanz besitzt, als eine homogene Linse von gleicher Gestalt und gleicher Brennweite, so dass es scheinen könnte, als seien schon durch das Vorstehende ihre periscopischen Vorzitge nachgewiesen. Schlüsse auf nicht homogene Linsen wären nur aus solchen Gleichungen gestattet, in welchen nur Cardinaldistanzen, und keine nnmittelbaren optischen Constanten der Linse vorkommen. Es gelingt aber nicht, aus den Gleichungen $73-76 \mathrm{r}$, $\varrho$ und $\mathrm{d}$, etwa dureh Einführung von $\mathrm{F}$ und $\Delta_{2}$, herauszuschaffen.

Die periscopischen Eigenschaften centrirter Systeme, insbesondere von der Art der Krystalllinse, werden vielmehr den Gegenstand einer weiteren Mittheilung bilden.

\section{Zusatz zum 1. Theile dieser Arbeit.}

Im ersten Theil (S. 449, 451) ist nachgewiesen worden, dass der geometrische Ort des zweiten Brennpuncts sowohl für eine einfache Fläche als für eine dünne Linse eine durch den centralen Brennpunct gehende Kugelfläche, ihr Schnitt in der Ebene der Zeichnung ein Kreis ist. Dieser Kreis ist jedoch nicht, wie S. 451 gesagt worden ist, identisch für die einfache Fläche und für die Linse von gleicher Brennweite. Die Zeichnung Fig. 2, Taf. VI ist nur für die Linse gültig; hier ist nämlich die Gleichung 13, welche sowohl für die Fläche wie für die Linse den Werth der zweiten Brennweiten darstellt,

$$
\mathrm{F}_{2}=\frac{\mathrm{nr}}{\mathrm{n}^{2}-1}\left(\sqrt{\mathrm{n}^{2}-\sin ^{2} \varphi}+\cos \varphi\right)
$$

zugleich excentrische Polargleichung eines Kreises, dessen Polarwinkel $=\varphi$ sind; sein Radius ist $\frac{n^{2} r}{n^{2}-1}=\frac{n F}{n+1}$, und die Excentricität des Pols gleich $\frac{\mathrm{n} \mathrm{r}}{\mathrm{n}^{2}-1}=\frac{\mathrm{F}}{\mathrm{n}+1}$. Für die einzelne Fläche dagegen ist diese Gleichung, wenn sie zugleich Polargleichung des Kreises der zweiten Brennpuncte sein soll, so umzuformen, dass die Brechungswinkel $\psi$ als Polarwinkel auftreten; sie nimmt dann die Form an

$$
\mathbf{F}_{2}=\frac{\mathrm{n}^{2} \mathrm{r}}{\mathrm{n}^{2}-1}\left(\sqrt{\frac{1}{\mathrm{n}^{2}}-\sin ^{2} \psi}+\cos \psi\right)
$$

dies ist die excentrische Polargleichung eines Kreises vom Radius 
Ueber Brechung b. schiefer Incidenz, m. besond. Berücksichtigung d. Auges. 387 $\frac{n r}{n^{2}-1}=\frac{F}{n+1}$, und von der Polexcentricität $\frac{n^{2} r}{n^{2}-1}=\frac{n F}{n+1}$; beide Grössen also umgekehrt wie früher. Ich gebe noch in Fig. 7 die Fig. 7.

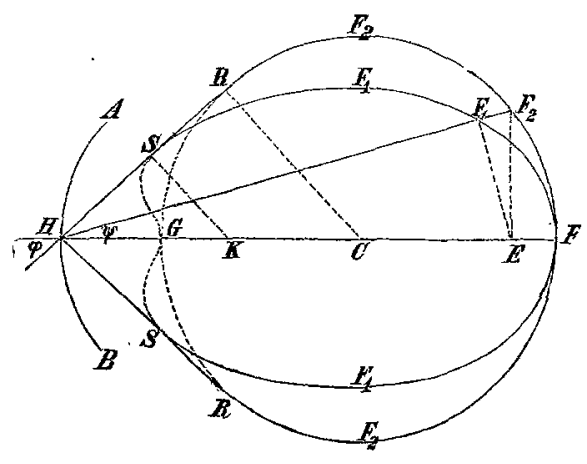

hieraus folgende Figur der Curven für die einfache brechende Fläche (und zwar für gleiche centrale Brennweite H F, wie sie in Fig. 2 des ersten Theils für die dünne Linse angenommen war). Die vom Incidenzpunct $\mathrm{H}$ an den Kreis $\mathrm{F}_{2} \mathrm{~F}_{2}$ gelegte Tangente $\mathrm{H} R$ ist zugleich der äusserste mögliche gebrochene Strahl (bei grösserem Incidenzwinkel findet totale Reflexion statt), und tangirt zugleich die Curve der ersten Brennpuncte $F_{1} F_{1}$ bei $S$; und zwar geht die Normale an den Punct $S$ durch den Krümmungsmittelpunct $K$ der brechenden Fläche. Die Beweise für die letzteren Sätze überlasse ich dem Leser.

Die in Fig. 5 des ersten Theils gegebene, einem Kreise sehr nahe kommende Curve, welche den Ort der zweiten Brennpuncte für die dicke Linse darstellt, lässt sich betrachten als eine Curve, deren Pol im Directionspunet liegt und sich mit diesem verschiebt, deren Vectoren aber gleich $\mathrm{F}_{2}+\psi_{2}$ sind; die Gleichıng 66 würde also die Polargleichung dieser Curve darstellen, wenn man von der Verschiebung des Poles absähe, und statt $\alpha$ den Polarwinkel $\varepsilon$ in die Gleichung einführte (mittels 40 oder 41 ). 